\title{
Optical and thermal restoration applied to thermo-elastic stress analysis by IR thermography
}

\author{
by S. Offermann, C. Bissieux and J. L. Beaudoin
}

Université de Reims, Unité de Thermique et Analyse Physique, Laboratoire d'Energétique et d'Optique, UFR Sciences, Moulin de la Housse, BP 1039, 51687 Reims Cedex 2, e-mail: jl.beaudoin@univ-reims.fr

\begin{abstract}
The resolution of stress analysis by means of infrared thermography is limited by the optical performance of the IR imaging equipment and by heat diffusion within the sample. The optical lowpass filtering is characterised by $1 \mathrm{D}$ and $2 \mathrm{D}$ transfer functions, while the $2 \mathrm{D}$ heat diffusion is modelled by a Bessel function. Iterative procedures are used for the restoration of both the optically smoothed contrasts and the thermally attenuated spatial gradients. The technique has been tested in some academic experiments, and then applied to stress maps of actual industrial parts.
\end{abstract}

\section{Introduction}

Thermoelastic stress analysis by means of radiometry is realised by measuring with an infrared radiometer the very small temperature variations of a sample under mechanical loading, then by calculating a stress map, using an adequate model of the thermoelastic coupling. The essential improvement of the signal to noise ratio can be realised as well by a "lock-in" detection [1, 2] as by a statistical treatment [3]. A high spatial resolution is desirable, but the quantitative evaluation of steep gradients is quite difficult, since they are attenuated by both the heat diffusion and the imaging process. So, the observed stress maps are smoothed relatively to the actual ones. Restoring the original stress maps needs to use some inverse techniques, based on the direct models of optical imaging and thermal effects.

Image formation operates as a low-pass filtering, which is characterised by a 2D response, usually known as the Point Spread Function (PSF), specific of each optical configuration.

On the other hand, the influence of thermal diffusion depends on the sample diffusivity and on the mechanical loading frequency. At the usual loading frequencies of industrial testings, the common assumption of adiabatic conditions is not satisfied and the heat diffusion effect must be taken into account [4].

The aim of the present work is to apply some iterative procedures, in order to solve both the optical and thermal inverse problems.

\section{Characterization of the IR camera by one dimensional response functions}

An idealised imaging system would give a strictly punctual image of a point source (Dirac's intensity distribution). However, due to residual optical aberrations, light diffraction phenomena and finite electronic band-width, modern systems produce images with broadened spatial distributions $\mathrm{D}\left(\mathrm{x}^{\prime}, \mathrm{y}^{\prime}\right)$. These distributions are called Point Spread Function (PSF) and can be more or less approximated by a Gaussian distribution:

$$
D\left(x^{\prime}, y^{\prime}\right)=e^{-\left(x^{\prime}+y^{\prime}\right)^{2} / \sigma^{2}}
$$

For a whole scene, the distribution of the image irradiance $I\left(x^{\prime}, y^{\prime}\right)$ can be expressed as the convolution of the object intensity $O(x, y)$ by the PSF, written here for a $1: 1$ magnification:

$$
I\left(x^{\prime}, y^{\prime}\right)=\iint O(x, y) \cdot D\left(x^{\prime}-x, y^{\prime}-y\right) \cdot d x \cdot d y
$$


This convolution integral turns to a simple algebraic product in the Fourier's space:

$$
T F(I)=T F(O) \cdot T F(D)
$$

Consequently, knowing the PSF D and measuring the image intensity I, an adequate inversion technique may allow for the calculation of the actual object intensity distribution $O$.

Unfortunately, measuring the $2 \mathrm{D}$ response PSF is somewhat intricate. The Line Spread Function (LSF), being the response to a 1D Dirac's distribution, can be measured more easily as the response to a very thin electrically heated wire. Figure 1 shows the LSF that we measured for an AGEMA 880 LW camera and three different configurations: without optical complement (full field width $14 \mathrm{~mm}$ ), with a $20^{\circ}$ lens (f.f. $w .: 13 \mathrm{~cm}$ ), with the $20^{\circ}$ lens and a $10 \mathrm{~mm}$ extension ring (f.f.w.: $35 \mathrm{~mm}$ ). The scanning time corresponding to a single line is about $280 \mu \mathrm{s}$. The experimental profiles have been fitted by some analytical functions. The LSF without optical complement is well described by a Gaussian function with a half-width $\sigma=6.18 \mu \mathrm{s}$. With the $20^{\circ}$ lens, the adequate functions are a Gaussian function with $\sigma=2.81 \mu$ s without extension ring, and $\sigma=3.47 \mu$ s with extension ring. Alternatively, the asymmetrical profile of the two LSF with the optical complement, may be taken into account by using two different functions for the right an left sides of each LSF. The right sides are Gauss functions ( $\sigma=2.73 \mu$ s and $2.81 \mu$ s respectively), while the left sides are taken as Voigt profiles $\left(\sigma^{\prime}=1.75 \mu\right.$ s and $2.57 \mu$ s).

To validate these analytical expressions of the LSF, we used them to calculate the Slit Response Functions (SRF). Indeed, we obtained a fairly good agreement between the calculated and experimentally determined SRF curves (figure 2). It can be seen that the use of asymmetric profiles yields very little benefit over the more approximate symmetric shapes.

Another confirmation was obtained from the image of a 1D grid with a variable spatial frequency. Figure 3 shows that the calculated and experimental images are quite similar, though the sampling period in the calculation was taken much smaller than in the experiment (1/140 line).

\section{Iterative optical restoration scheme or pseudo-inversion}

For solving the inverse optical problem, we have tested an iterative technique or pseudoinversion $[5,6]$. The actual unknown object intensity $O(x, y)$ generates an observed image $I_{0}(x, y)$. Firstly, this image is taken as a "virtual" object $O_{1}(x, y)$. The convolution of $O_{1}(x, y)$ by the low-pass filtering PSF $D(x, y)$ generates a new image $l_{1}(x, y)$ which is obviously less contrasted than $I_{0}(x, y)$. Then we determine a new virtual object $\mathrm{O}_{2}(x, y)$ which is a better approximation of $\mathrm{O}(\mathrm{x}, \mathrm{y})$ than $\mathrm{O}_{1}(\mathrm{x}, \mathrm{y})$ by the difference algorithm:

$$
O_{2}(x, y)=O_{1}(x, y)+\left[O_{1}(x, y)-I_{1}(x, y)\right]=2 O_{1}(x, y)-I_{1}(x, y)
$$

We repeat the procedure until the $n$-th calculated image $\ln (x, y)$ converges to the measured one $I_{0}(x, y)$. The corresponding object $O_{n}(x, y)$ should be a smart approach of the actual object $O(x, y)$. The convolutions are realised via the product of discrete Fourier transforms; for which we made use of the MathematicaTM built-in functions for the direct and inverse transforms.

Figure 4 shows the result of this iterative procedure on the one dimensional grid. The restored image clearly resembles more to the object than the untreated image. However, no miracle was to be expected. The highest spatial frequencies, having been too heavily filtered by the PSF, are not restored by this procedure. Nevertheless, due to the simplicity of the implementation, it appears as a valuable compromise.

\section{Harmonic thermal diffusion}

A similar pseudo-inversion technique has been used in order to restore the image of the thermoelastic heat sources, which are low-pass filtered by the heat diffusion. The feasibility of such a restoration by using the finite element method for the direct heat conduction model 
http://dx.doi.org/10.21611/qirt.1998.019

has already been proved [4]. A more simple approach in the case of periodically time-varying plane stresses, consists in expressing the temperature distribution $T(x, y)$ as a convolution of the heat sources distribution $q(x, y)$ by a proper "thermal harmonic point spread function". Previously we have defined such a THPSF $H\left(\vec{r} \mid \vec{r}^{\prime}\right)$ as solution of the heat diffusion equation, where the heat source is expressed as a spatial Dirac's delta function [7]:

$$
\nabla^{2} H\left(\vec{r} \mid \vec{r}^{\prime}\right)-\frac{j \omega}{a} H\left(\vec{r} \mid \vec{r}^{\prime}\right)=-\frac{\delta\left(\vec{r}-\vec{r}^{\prime}\right)}{k}
$$

where $\omega$ is the frequency, $k$ is the thermal conducitvity and a the thermal diffusivity.

Generally, such a transfer function is related to the corresponding Green's function by:

$$
H\left(\vec{r} \mid \vec{r}^{\prime}\right)=\frac{a}{k} \int_{0}^{\infty} G\left(\vec{r} \mid \vec{r}^{\prime}, \tau\right) e^{-j \omega \tau} d \tau
$$

In many cases, these functions are available in the literature [8] and the solution of the heat diffusion equation in harmonic regime can then be obtained from the integration over the source field:

$$
T(\vec{r})=\iiint H\left(\vec{r} \mid \vec{r}^{\prime}\right) q\left(\vec{r}^{\prime}\right) d R
$$

In cartesian $x-y$ geometry and for an infinite medium, the THPSF is a Bessel function of the second kind:

$$
H_{\infty}\left(x, y \mid x^{\prime}, y^{\prime}\right)=\frac{1}{2 \pi k} K_{0}\left(\sigma \sqrt{\left(x-x^{\prime}\right)^{2}+\left(y-y^{\prime}\right)^{2}}\right) \text { with } \sigma=\sqrt{\frac{j \omega}{a}}
$$

Then, the integral expression of the temperature becomes a convolution integral:

$$
T(x, y)=\int_{-\infty}^{+\infty} \int_{-\infty}^{+\infty} H\left(x, y \mid x^{\prime}, y^{\prime}\right) q\left(x^{\prime}, y^{\prime}\right) d x^{\prime} d y^{\prime}=H(x, y) \otimes q(x, y)
$$

For finite-size samples with zero flux at the boundaries, the image THPSF $H_{\text {im }}$ must be added. Then, a suitable change of variables allows to obtain a convolution again:

$$
T(x, y)=\left(H_{\infty}(x, y)+H_{i m}(x, y)\right) \otimes q(x, y)=H_{\infty}(x, y) \otimes\left(q(x, y)+q_{i m}(x, y)\right)
$$

\section{Restoration of thermoelastic heat sources}

The thermoelastic coupling introduces a heat source term $q(x, y, t)$ into the heat equation:

$$
\rho C_{\sigma} \frac{d T(x, y, t)}{d t}-k \nabla^{2} T(x, y, t)=q(x, y, t)=-\alpha T_{0} \frac{d I_{1}(x, y, t)}{d t}
$$

where $I_{1}$ is the sum of the principal stresses, $\alpha$ the 1-D expansion coefficient, $T_{0}$ the average temperature. In the harmonic case:

$$
\nabla^{2} T(x, y)-\frac{j \omega}{a} T(x, y)=-\frac{q(x, y)}{k}=\frac{\alpha T_{0} j \omega I_{1}(x, y)}{k}
$$

The temperature spatial distribution $T(x, y)$ is proportional to the stress spatial distribution, only if the effect of heat diffusion in the medium can be neglected. This is not the case at too low frequencies, and/or high conductivity, and/or steep temperature gradients. 
However, with the direct heat diffusion model (eq. 10), the thermally attenuated spatial contrasts can be restored by an iterative scheme, similar to that used for the optical restoration. This method intends to reconstruct here the source distribution $q_{0}(x, y)$, which would yield the temperature distribution $T_{a 0}(x, y)$, the modulus of which arises from the optical restoration. A first virtual source distribution is calculated, using the adiabatic relationship:

$$
q_{1}(x, y)=\rho C_{\sigma} \omega\left|T_{a 0}(x, y)\right|
$$

Then, convoluting by multiplication in the Fourier space, the direct conduction model is applied to this source distribution, yielding a temperature distribution $T_{a 1}(x, y)$, which modulus is even less contrasted than that of $T_{a 0}(x, y)$. Analogously to the optical restoration, the difference algorithm is repeated ( 4 to 5 times) until convergence:

$$
q_{2}(x, y)=2 q_{1}(x, y)-\rho C_{\sigma} \omega\left|T_{1}(x, y)\right|
$$

The restoration techniques presented above have been applied to several automotive parts. We present here the results concerning a motor crank bar, made from G.S. cast iron. It was subjected to a harmonic uniaxial tensile loading of $10 \mathrm{kN}$ by a SCHENK fatigue machine, at a frequency of $30 \mathrm{~Hz}$. The IR camera had a $20^{\circ}$ lens and a $10 \mathrm{~mm}$ extension tube. The optical restoration was first applied to the image as just obtained after the noise reduction procedure. For this, we simply built a 2D PSF by the product of the ID symmetric LSF along two orthogonal directions. This optical restoration had only a very slight effect.

We concluded that the spatial gradients were not smoothed here by the optical response, since the thermal filtering acted physically at first and more strongly than the optical filtering. So, any information of higher spatial frequencies, when compared to the width of the PSF Fourier transform, can here be considered as noise. Consequently, we chose this PSF transform as a low-pass filter before the restoration of the thermal effect.

Figure 5 and figure 6 present the stress map before and after correction of the heat diffusion effect. Though no spectacular changes are observed, it seems that the adiabatic approximation leads to at least $10 \%$ underestimation of the values of the stress concentrations.

\section{Conclusion}

The complete problem of thermoelastic stress analysis by means of infrared thermography consists in the quantitative evaluation of a stress field from a series of infrared camera images. A time noise rejection procedure is first applied to this series, yielding a stress map, which remains altered by different physical low-pass filters. The optical and thermal filters have been characterized by their response functions, namely the PSF and THPSF. An inversion procedure, iterating the convolution of the map by the filter, brings a reasonable enhancement of the spatial contrasts. Practically, only the effect of the stronger filter needs to be restored, while the weaker one provides a relevant residual noise rejection filter.

\section{REFERENCES}

[1] MOUNTAIN (D. S.) and WEBBER (J. M. B.) - Stress Pattern Analysis by Thermal Emission (SPATE). SPIE Proc.167, 1978, p. 189-196.

[2] BREMONT (P.) - La Thermographie Infrarouge pour voir les contraintes. Mesures, Vol. 673 , mars 1995, p. 53-56. [in French]

[3] OFFERMANN (S.), BISSIEUX (C.), BEAUDOIN (J L.) - Thermoelastic stress analysis with standard Thermographic Equipment by means of statistical noise rejection. Research in Non destructive Evaluation, Vol. 7, Nr. 4, 1996, p. 239-251.

[4] OFFERMANN (S.), BISSIEUX (C.), BEAUDOIN (J. L.), FRICK (H.) - Thermoelastic stress analysis under nonadiabatic conditions. Exp. Mechanics, Vol. 37, Nr. 4, December 1997. 
http://dx.doi.org/10.21611/qirt.1998.019

[5] BIJAOUI (A.). - Image and Information - Introduction to numerical processing of images. Masson, Paris 1984

[6] ARGIRIOU (A.) - La mesure en Thermographie Infrarouge, calibration et traitement du signal... Ph.D. Thesis, Université de Provence, 1987 [in French]

[7] GILLET (Y.), OFFERMANN (S.), BISSIEUX (C.), BEAUDOIN (J. L.) - Thermomechanical modelling by harmonic response functions, Conf. Advanced Concepts and Techniques in Thermal Modelling (Eurotherm N ${ }^{\circ} 51$ ), Mons (Belgium), October 1997.

[8] BUTKOVSKIY (A. G.) - Green's functions and transfer functions handbook, Wiley, New York, 1982.

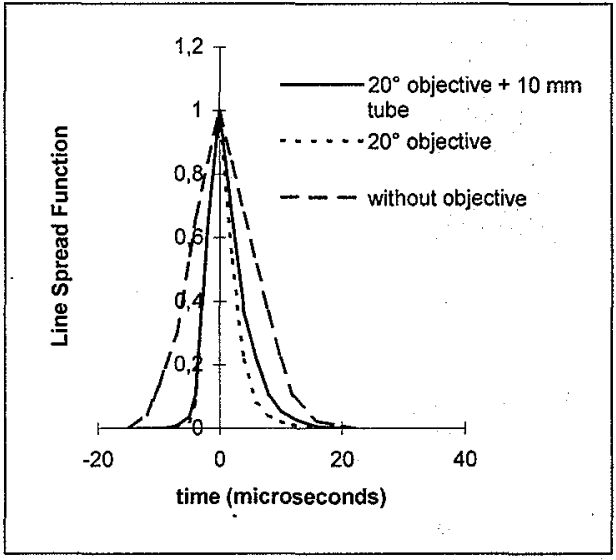

Fig 1. Horizontal line spread functions for different optical configurations

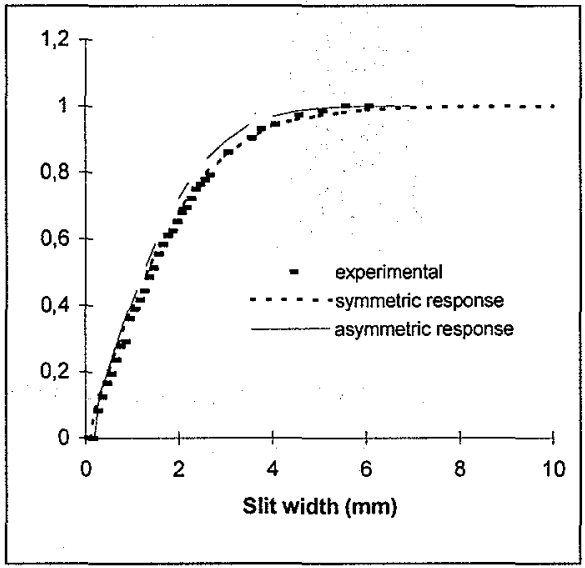

Fig. 2. Experimental and theoretical SRF for the $20^{\circ}$ objective

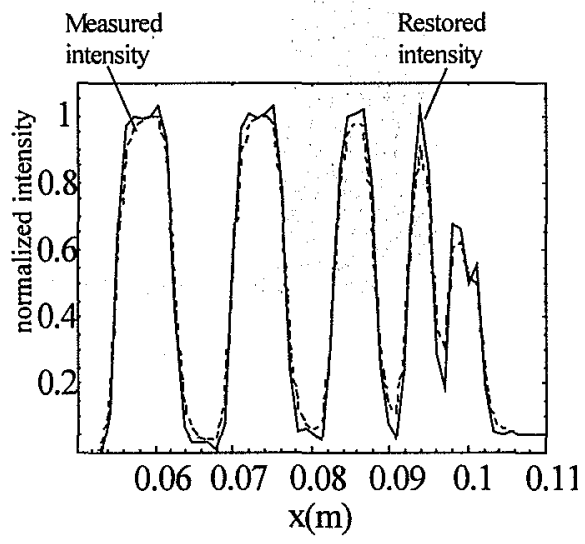

Fig. 4. Restoration of the attenuated contrasts
Fig. 3. Calculated and measured attenuation of thermal contrasts
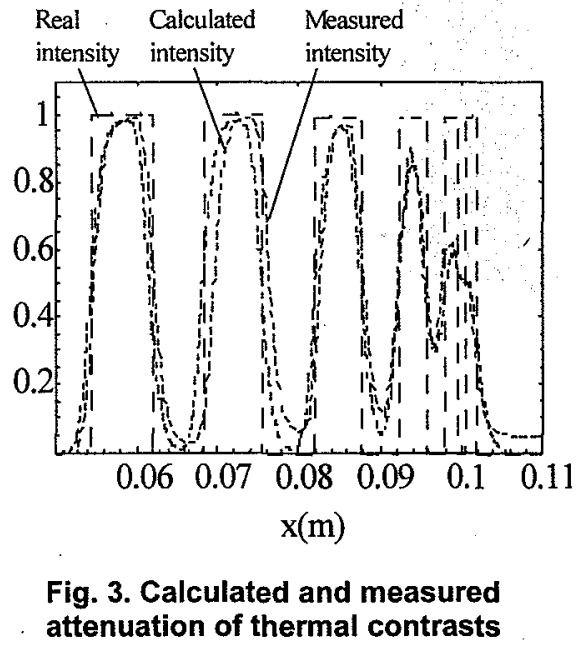
http://dx.doi.org/10.21611/qirt.1998.019

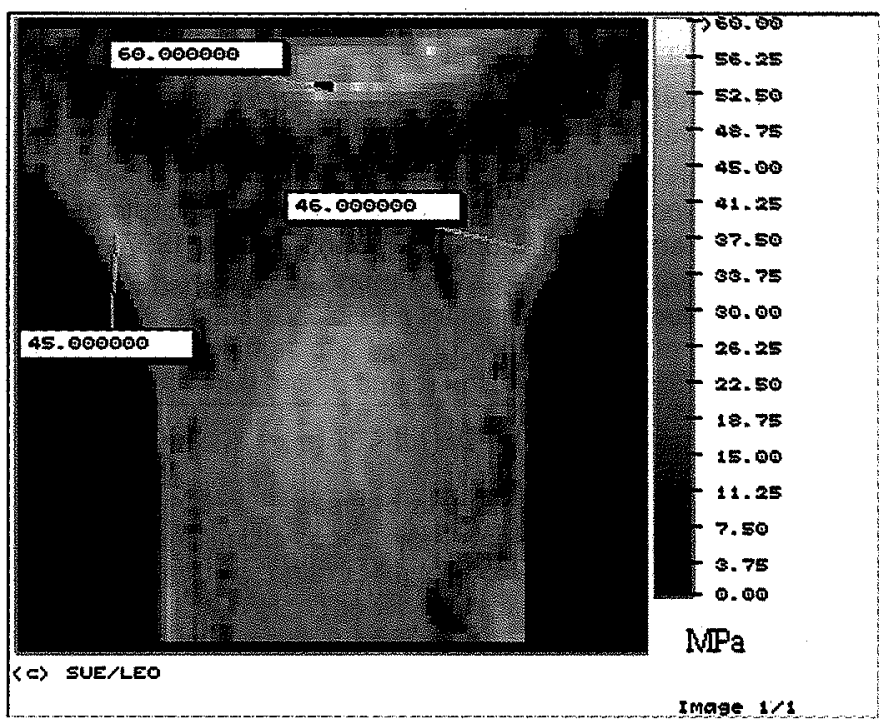

Fig. 5. Experimental stress map

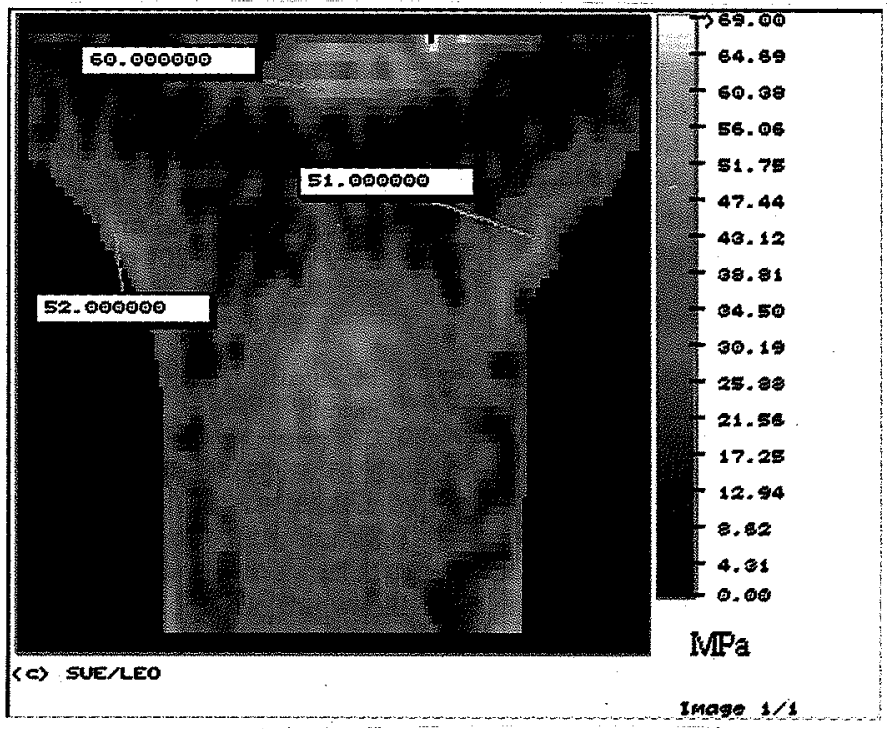

Fig. 6. Restored stress map 